\title{
Gastroenterology Training in Japan
}

\author{
Hisashi Hatanaka $^{1}$ - Hironori Yamamoto ${ }^{1}$ Alan Kawarai Lefor ${ }^{2}$. \\ Kentaro Sugano ${ }^{3}$
}

Received: 17 December 2015/ Accepted: 26 January 2016/Published online: 10 February 2016

(C) Springer Science+Business Media New York 2016

\section{Introduction}

Jichi Medical University (JMU) was established in 1972 with a stated mission to train general practitioners as highly capable health care providers in rural Japan. JMU has a gastroenterology curriculum for junior residents and gastroenterology fellows and is approved as a teaching institution for board-certified gastroenterologists by the Japanese Society of Gastroenterology (JSGE). The JSGE is the largest society of gastroenterologists in Japan, with more than 34,000 members in 2015 and is the governing body in Japan that certifies gastroenterology programs. The JSGE has offered subspecialty training in gastroenterology since 1988. In 2013 with the guidance of Professor Deborah D. Proctor from the Education and Training Committee of the American Gastroenterological Association [1, 2], the gastroenterology leadership in Japan revised our training program curriculum to provide competency-based education (CBE). At that time, we introduced an educational model for gastroenterology education for medical students and trainees in Japan.

Hisashi Hatanaka

hatanaka@jichi.ac.jp

1 Division of Gastroenterology, Department of Medicine, Jichi Medical University, 3311-1 Yakushiji, Shimotsuke, Tochigi 329-0498, Japan

2 Department of Surgery, Jichi Medial University, Shimotsuke, Japan

3 Jichi Medical University, Shimotsuke, Japan

\section{Time Line: Medical Student to Resident to Gastroenterology Fellowship}

Japanese medical education is 6 years. During the first 1-2 years, students study liberal arts and basic medical science. Over the next 2 years, students study pathophysiology, diagnostics, environmental medicine, and emergency medicine. At this time, students are required to pass the tests to determine whether or not their basic skills on physical examination and general clinical knowledge are satisfactory to participate in actual clinical care teams as student doctors.

In their last 2 years of medical school, medical students participate in clinical teams, and study physical examination and the diagnostic approach for their patients. In the gastroenterology department, students participate in endoscopic examinations and treatment for their patients, including endoscopic submucosal dissection, endoscopic hemostasis, and endoscopic biliary drainage in gastroenterology either directly or indirectly as assistants or observers. Medical students receive lectures regarding the stomach, colon, liver, pancreas, biliary tract, and abdominal ultrasonography (US) during a two-week rotation in gastroenterology. Students who are especially interested in gastroenterology attend a digestive disease seminar. Themes at the monthly seminar include upper gastrointestinal endoscopy, colonoscopy, abdominal US, US-guided biliary drainage, interpretation of abdominal CT scans, endoscopic retrograde cholangiopancreatography (ERCP), and related procedures. Medical students are required to pass the Japanese National Medical License Examination in order to begin their residency training. Graduates are then sent to one of 47 prefectures where their postgraduate educations are continued in their assigned teaching hospitals. 
All residents must have at least 2 years of clinical training under board-certified physicians in accredited training hospitals designated by the Japanese Ministry of Health, Labor and Welfare and the Japanese Society of Internal Medicine. The committee of Japan Residency Matching Program (JRMP) matches training hospitals with residents. After the 2 years of junior residency (clinical training), those who want subspecialize in gastroenterology need to be trained in a hospital that is approved by the JSGE. Minimum requirements for a JSGE-qualified training hospital include the following: a minimum of 30 beds dedicated to gastroenterology, one board-certified instructor, and two additional board-certified gastroenterologists.

Residents rotate in various departments during their 2 years of junior residency. Most residents in the internal medicine program have training in gastroenterology for 3 months at JMU. A bedside team usually consists of three doctors (one resident and two directors). One of the two directors is a gastroenterology fellow [postgraduate year (PGY) 4-7], and another director is a gastroenterology subspecialist (PGY-8 or more). Patients seen in the department have various diseases of the gastrointestinal tract (e.g., gastric cancer, gastroduodenal ulcers, gastroesophageal varices, intestinal bleeding, colon cancer, inflammatory bowel disease), liver (e.g., hepatocellular carcinoma, liver cirrhosis, acute hepatitis), biliary tract (e.g., biliary tract cancer, acute cholangitis, cholecystitis, choledocholithiasis), and pancreas (e.g., pancreatic cancer, acute pancreatitis).

Residents perform physical examinations and are responsible for the care of 8-10 inpatients. Clinical teams conduct patient rounds at least twice daily and confirm the clinical condition of the patients. At least two directors on the same team repeatedly check the diagnostic and treatment plans made by the residents.

Our department also gives residents an opportunity to perform follow-up endoscopic examinations on their patients under the supervision of the gastroenterology directors. Residents receive endoscopic training using an upper gastrointestinal simulator under the guidance of a gastroenterology fellow before performing the procedure on patients (Fig. 1). Residents provide emergency care twice a month under the guidance of senior physicians.

To pursue training in a subspecialty, residents must first obtain certification in a basic medical specialty in Japan such as internal medicine, surgery, radiology, or pediatrics (Table 1). Certification for internal medicine involves submitting 20 representative case reports encompassing various subspecialty areas (cardiology, gastroenterology, pulmonary medicine, nephrology, hematology, neurology, rheumatology, infectious diseases, and emergency care) during their subspecialty rotation followed by passing a written examination.
Gastroenterology fellowship training involves attending outpatient clinic and performing endoscopic procedures. After performing about 100 upper gastrointestinal endoscopies, they receive training in colonoscopy. Acquisition of the skills needed for colonic endoscopic mucosal resection and hemostasis permits moving to the next steps, such as ERCP. Gastroenterology fellows also train in performing and interpreting abdominal ultrasounds as in Japan abdominal ultrasounds are performed by the gastroenterologists rather than by radiologists. Fellows become a board-certified gastroenterologist of the JSGE after completion of the defined curriculum set by the JSGE [1]. This curriculum has a program of competency-based education including evaluations of capability in multiple domains including specialized medical knowledge and technique, doctor-patient relationship, cooperation with medical staffs, safety for the patients and doctors, medical ethics, and professionalism. Certification is given by the JSGE after successfully meeting specific requirements and passing a certifying examination.

After obtaining certification as a specialist, recertification is needed every 5 years by acquiring the necessary points for continuous medical education (CME). CME points are granted for attendance and/or presentation of papers at a congress, but at least one attendance to a whole day postgraduate seminar is mandatory. There are no recertification examinations required.

Becoming a board-certified endoscopist of the Japanese Gastroenterological Endoscopy Society (JGES) requires extensive endoscopic experience (e.g., more than 500 upper gastrointestinal endoscopies, 250 colonoscopies, and 20 endoscopic treatments) over the previous 5 years.

In Japan, The Japan Society of Hepatology (JSH) has their own subspecialty (Hepatology) certification program independent from the JSGE.

\section{Future Directions}

Issues for clinical training of gastroenterologists in Japan are to integrate competency-based education requirements into the gastroenterology training program. Most senior physicians involved in resident education have not received training in competency-based educational methods. In Japan, the traditional way of teaching was subjective- the trainee learned how to evaluate and manage patients by modeling the behavior of their senior attendings with otherwise minimal interaction between trainee and teacher. Thus, future endeavors to improve gastroenterology training include the integration of e-learning systems, simulators, and endoscopic hands-on training and more thorough incorporation of competency-based educational methods. 


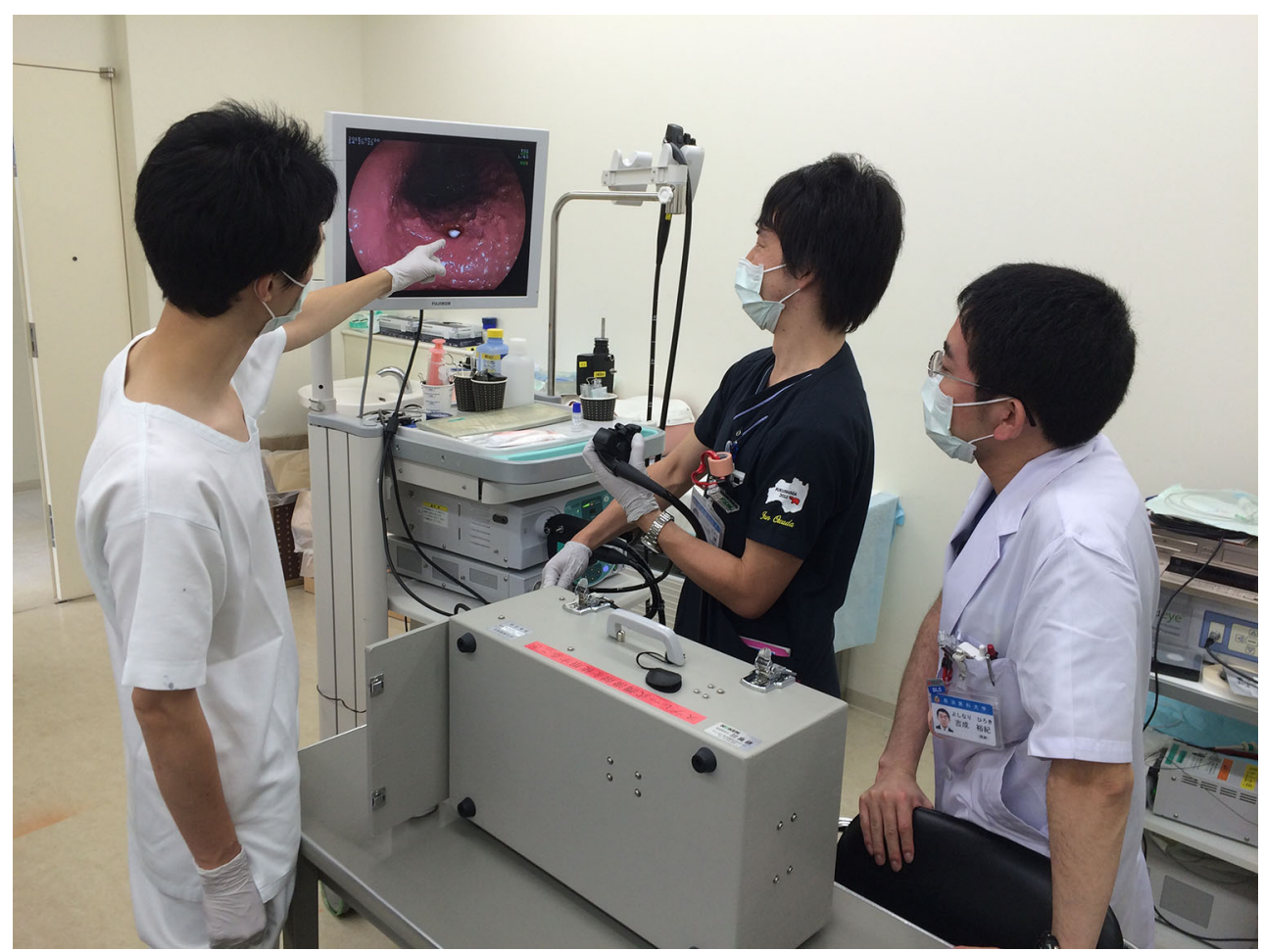

Fig. 1 Junior residents receive endoscopic training using an upper gastrointestinal simulator under the guidance of a gastroenterology fellow

Table 1 Subspecialty training in gastroenterology in Japan

\begin{tabular}{llll}
\hline Qualifying examination & Training level & Training course & Duration \\
\hline National Medical License Examination & Medical student & Medical school & 6 years \\
& Resident & Residency & $>3$ years $(*>5$ years) \\
Basic medical specialty & Specialist in Internal Medicine & GI fellowship & $>3$ years $(*>2$ years) \\
& Surgery/Radiology/Pediatrics & & Recertify every 5 years \\
Board-certified gastroenterologist of JSGE & Specialist in Gastroenterology & \\
Endoscopist of JGES & Endoscopy & \\
Hepatolologist of JSH & Hepatology & \\
\hline
\end{tabular}

JSGE Japanese Society of Gastroenterology, JGES Japan Gastroenterological Endoscopy Society, JSH Japan Society of Hepatology, GI gastroenterology

* In case of specialist in Surgery/Radiology/Pediatrics training level

\section{References}

1. Subspecialty council committee of Japan Society of Gastroenterology. The training curriculum of Japanese Society of Gastroenterology 2013. Yamaguchi, Toyo Tosho; 2013 (in Japanese).
2. Sugano K. Renovation of subspecialty program for gastroenterology in Japan. Nihon Syoukakibyo Gakkai Zasshi (J Jpn Soc Gastroenterol). 2012;109:9-14 (in Japanese with English abstract). 